\title{
Therapeutic Role of Ricinus Communis L. Root Bioactive Compounds in Rheumatoid Arthritis
}

\author{
Priyanka Pandey \& Sugandha Tiwari \\ DGPG College, Kanpur.
}

Received: 20 Oct 2020 / Accepted: 18 Nov 2020/ Published online: 01 Jan 2021

*Corresponding Author Email:

\begin{abstract}
Ricinus communis L. is commonly called castor plant. The plant belongs to family Euphorbiaceae. Plant has a great medicinal value. Each part of the plant is used in the treatment of different disorders. The roots of Ricinus communis are widely used in the treatment of different diseases from the very ancient time. Roots of castor plant are used in the treatment of rheumatism, fever and inflammation etc. In the present work phytochemical profiling of Ricinus was done through GC-MS analysis. The phytochemical profiling of castor root reveals the presence of different active phytochemicals. Preliminary physicochemical analysis shows total ash content 5.21\%, acid insoluble ash $91 \%$, water soluble ash content $10 \%$. Phytochemical analysis reveals the presence of carbohydrate, protein, phenols, saponins. For the GC-MS profiling of Ricinus two water and methanol extracts were used. Methanol extracts shows more 78 compounds while water based extracts shows 6 compounds.
\end{abstract}

\section{Keywords}

Ricinus communis, GCMS, phytochemicals.

\section{INTRODUCTION:}

In present time people have started to be looking for ancient medicinal system for the treatment of disease as these systems have no side effects, effective and are safe. According to the ancient Indian literature all plant parts have medicinal substances ${ }^{1}$. In India, pharmacological and phytochemical investigation has been carried out only on a very small population of wild plants ${ }^{2}$. Wild plants are the major source of the herbal drugs for the local people and also for the herbal drug industries. Normally the raw material purchased from the market is adultarated ${ }^{3}$. Adulteration and substitution of raw material is a global problem for local people and herbal drug industries. ${ }^{4}$ in present study, Ricinus root was selected to authenticate it based on physicochemical, preliminary phytochemical analysis and chemical profiling through GCMS.

Botanical name of castor is Ricinus communis $\mathrm{L}$. 'Eranda' is a Sanskrit name of castor ${ }^{5}$. Castor belongs to the family Euphorbiaceae. It is a wild flowering plant grow throughout the world. Castor is a fast growing shrub or sometime soft wooded small tree of about 3-5 meter hight. Leaves are green, large 20$25 \mathrm{~cm}$ in length and $10-17 \mathrm{~cm}$ in breath, petiolate, palmately lobed, 5-7 lobes with serrate margins. Different parts of the plant like root, stem, leaves, seed oil are used in the treatment of inflammatory and liver disorders ${ }^{6}$. Root of Ricinus is sweet in taste and it is used in the treatment of different disorders ${ }^{7}$. In ayurveda, castor roots are used in the treatment of rheumatism, liver disorders, fever, backache etc ${ }^{8}$. The extracts from different parts of the plant shows 
analgesic, diuretic, anti-asthmatic, anthelmintic and many other medicinal properties ${ }^{9-14}$.

\section{MATERIAL AND METHOD:}

Fresh roots of Ricinus communis $\mathrm{L}$. are collected from the different parts of Kanpur U.P. in the month of December and authenticated by Dr. Sugandha Tiwari, Professor, D.G.P.G. College Kanpur. Roots are washed properly, shade dried, powdered in the mechanical grinder. Powdered material is preserved in air tight glass bottles for further use.

Physicochemical study-Ash content, water soluble ash, acid insoluble ash, extractive values are determined according to well-known methods and procedure ${ }^{15,16,17}$.

\section{Preliminary phytochemical profiling}

\section{Extract preparation}

The crude drug powder is subjected for the preparation of methanolic and aqueous extract .10 grams of root powder was used for extraction with $200 \mathrm{ml}$ methanol, shaken vigorously for 5-10 min and left for $24 \mathrm{~h}$. after which the extract is filtered. Water extract was also prepared by using the same method.100 $\mathrm{ml}$ of both the extracts were evaporated to dryness in water bath. Dry extracts were cooled and weighted. Percentage yield (extractive value) was calculated. Remaining $100 \mathrm{ml}$ water and methanolic extract were used for the preliminary phytochemical testing according to the standard procedure $^{18}$

\section{GCMS analysis}

GC-MS analysis was done by Indian Institute of Science Education and Research, Bhopal. GCMS analysis was done by Agilent 7890 A GC with5975 CMS system, an oven temperature from $50^{\circ} \mathrm{C}$ to $280^{\circ} \mathrm{Cat} 4^{\circ} \mathrm{C} / \mathrm{min}$ and held at this temperature for 5 min; inlet and interface temperatures were $250^{\circ} \mathrm{C}$ and $280^{\circ} \mathrm{C}$, respectively. Carrier gas was $\mathrm{He}$ at a flow rate of $1.0 \mathrm{ml} / \mathrm{min}$ (constant flow). $0.2 \mathrm{ml}$ of sample was injected under split of 20:1. EIMS: electron energy, $70 \mathrm{eV}$. Interpretation of mass spectrum GCMS was conducted using data base of NIST, having more than 62,000 patterns. The spectrum of the known compounds was compared with the NIST library.

\section{RESULT AND DISCUSSION:}

Physicochemical analysis

Results of physicochemical analysis were mentioned in table-1(ash value) and table-2(extractive value)

Table -1 ash content in Ricinus communis root

\begin{tabular}{ll}
\hline Type of ash & Values in \% w/w \\
\hline Total ash content & $5.20 \%$ \\
Acid insoluble ash & $0.91 \%$ \\
Water soluble & $10.80 \%$ \\
\hline
\end{tabular}

Table-2 Extractive values

\begin{tabular}{ll}
\hline Solvent & Extractive values \% W/W \\
\hline Water & 10.4 \\
Methanol & 8.6 \\
\hline
\end{tabular}

Phytochemical analysis- results of preliminary phytochemical analysis of crude drug for the presence or absence of different phytochemicals like alkaloids, tannins and saponins etc. are shown in the table -3 .

Table-3 preliminary phytochemical test of Ricinus communis root

\begin{tabular}{lll}
\hline Phytochemical test & Water extract & Methanolic extract \\
\hline Alkaloid & + & + \\
Carbohydrates & + & + \\
Saponins & + & + \\
Flavonoids & + & + \\
Terpenoids & + & + \\
Protein & - & - \\
\hline & +present, -absent
\end{tabular}


Table 4: GCMS Profiling of methanolic extract of Ricinus root.

\begin{tabular}{|c|c|c|c|c|}
\hline SI.No & Compound & Chem. formula & Mol. wt & Prob. \\
\hline 1. & 2-Methoxy-4-vinylphenol & $\mathrm{C}_{9} \mathrm{H}_{10} \mathrm{O}_{2}$ & 150 & $28.4 \%$ \\
\hline 2. & Ethanone, 1-(2-hydroxy-5-methylphenyl)- & $\mathrm{C}_{9} \mathrm{H}_{10} \mathrm{O}_{2}$ & 150 & $21.8 \%$ \\
\hline 3. & 4-Hydroxy-2-methylacetophenone & $\mathrm{C}_{9} \mathrm{H}_{10} \mathrm{O}_{2}$ & 150 & $9.92 \%$ \\
\hline 4. & 4-Hydroxy-3-methylacetophenone & $\mathrm{C}_{9} \mathrm{H}_{10} \mathrm{O}_{2}$ & 150 & $7.60 \%$ \\
\hline 5. & Ethanone, 1-(3-methoxyphenyl)- & $\mathrm{C}_{9} \mathrm{H}_{10} \mathrm{O}_{2}$ & 150 & $5.97 \%$ \\
\hline 6. & 1-(4-Hydroxymethylphenyl)ethanone & $\mathrm{C}_{9} \mathrm{H}_{10} \mathrm{O}_{2}$ & 150 & $3.86 \%$ \\
\hline 7. & Sucrose & $\mathrm{C}_{12} \mathrm{H}_{22} \mathrm{O}_{11}$ & 342 & $12.6 \%$ \\
\hline 8. & d-Glycero-d-ido-heptose & $\mathrm{C}_{7} \mathrm{H}_{14} \mathrm{O}_{7}$ & 210 & $9.89 \%$ \\
\hline 9. & d-Glycero-d-galacto-heptose & $\mathrm{C}_{7} \mathrm{H}_{14} \mathrm{O}_{7}$ & 210 & $5.69 \%$ \\
\hline 10. & Galacto-heptulose & $\mathrm{C}_{7} \mathrm{H}_{14} \mathrm{O}_{7}$ & 210 & $4.81 \%$ \\
\hline 11. & Lactose & $\mathrm{C}_{12} \mathrm{H}_{22} \mathrm{O}_{11}$ & 342 & $4.25 \%$ \\
\hline 12. & $\begin{array}{l}\alpha-D-G l u c o p y r a n o s i d e, ~ O-\alpha-D-g l u c o p y r a n o s y l-(1 . f w d a r w .3)-\beta-D- \\
\text { fructofuranosyl }\end{array}$ & $\mathrm{C}_{18} \mathrm{H}_{32} \mathrm{O}_{16}$ & 504 & $4.25 \%$ \\
\hline 13. & Hexadecanoic acid, methyl ester & $\mathrm{C}_{17} \mathrm{H}_{34} \mathrm{O}_{2}$ & 270 & $77.8 \%$ \\
\hline 14. & Pentadecanoic acid, 14-methyl-, methyl ester & $\mathrm{C}_{17} \mathrm{H}_{34} \mathrm{O}_{2}$ & 270 & $13.0 \%$ \\
\hline 15. & Pentadecanoic acid, 13-methyl-, methyl ester & $\mathrm{C}_{17} \mathrm{H}_{34} \mathrm{O}_{2}$ & 270 & $3.12 \%$ \\
\hline 16. & Hexadecanoic acid, 2-methyl- & $\mathrm{C}_{17} \mathrm{H}_{34} \mathrm{O}_{2}$ & 270 & $3.00 \%$ \\
\hline 17. & Pentadecanoic acid, methyl ester & $\mathrm{C}_{16} \mathrm{H}_{32} \mathrm{O}_{2}$ & 256 & $0.68 \%$ \\
\hline 18. & Hexadecanoic acid, 15-methyl-, methyl Ester & $\mathrm{C}_{18} \mathrm{H}_{36} \mathrm{O}_{2}$ & 284 & $0.57 \%$ \\
\hline 19. & n-Hexadecanoic acid & $\mathrm{C}_{16} \mathrm{H}_{32} \mathrm{O}_{2}$ & 256 & $69.3 \%$ \\
\hline 20. & I-(+)-Ascorbic acid 2,6-dihexadecanoate & $\mathrm{C}_{38} \mathrm{H}_{68} \mathrm{O}_{8}$ & 652 & $18.5 \%$ \\
\hline 21. & Palmitic anhydride & $\mathrm{C}_{32} \mathrm{H}_{62} \mathrm{O}_{3}$ & 494 & $3.76 \%$ \\
\hline 22. & Isopropyl Palmitate & $\mathrm{C}_{19} \mathrm{H}_{38} \mathrm{O}_{2}$ & 298 & $2.05 \%$ \\
\hline 23. & i-Propyl 14-methyl-pentadecanoate & $\mathrm{C}_{19} \mathrm{H}_{38} \mathrm{O}_{2}$ & 298 & $1.12 \%$ \\
\hline 24. & i-Propyl hexadecanoate & $\mathrm{C}_{19} \mathrm{H}_{38} \mathrm{O}_{2}$ & 298 & $0.76 \%$ \\
\hline 25. & Pregn-5-en-20-one, 3-(acetyloxy)-17-hydroxy-, (3ß)- & $\mathrm{C}_{23} \mathrm{H}_{34} \mathrm{O}_{4}$ & 374 & $24.8 \%$ \\
\hline 26. & $\begin{array}{l}\text { Cyclopenta[d]anthracene-8,11-dione, 1,2,3,3a,4,5,6,6a,7,8,11,12- } \\
\text { dodecahydro-3-(1-methylethyl)-12-hydroxy- }\end{array}$ & $\mathrm{C}_{20} \mathrm{H}_{26} \mathrm{O}_{3}$ & 314 & $16.0 \%$ \\
\hline 27. & 5,16,20-Pregnatriene-3beta,20-diol diacetate & $\mathrm{C}_{25} \mathrm{H}_{34} \mathrm{O}_{4}$ & 398 & $5.36 \%$ \\
\hline 28. & $\begin{array}{l}\text { 17Alpha-ethynyl-6beta-methoxy-3alpha,5-cyclo-5alpha- } \\
\text { androstane-17beta,19-diol }\end{array}$ & $\mathrm{C}_{22} \mathrm{H}_{32} \mathrm{O}_{3}$ & 344 & $2.76 \%$ \\
\hline 29. & $\begin{array}{l}(5 \beta, 13 \beta) \text { Androst-8-en-3-one, 17-19-diacetoxy-4,4-dimethyl } \\
\text { 1H-Cyclopropa[3,4]benz[1,2-e]azulene-5,7b,9,9a-tetrol, }\end{array}$ & $\mathrm{C}_{25} \mathrm{H}_{36} \mathrm{O}_{5}$ & 416 & $2.65 \%$ \\
\hline 30. & $\begin{array}{l}\text { 1a,1b,4,4a,5,7a,8,9-octahydro-3-(hydroxymethyl)-1,1,6,8- } \\
\text { tetramethyl- }\end{array}$ & $\mathrm{C}_{26} \mathrm{H}_{36} \mathrm{O}_{8}$ & 476 & $2.55 \%$ \\
\hline 31. & trans-Dehydroandrosterone, methyl ether & $\mathrm{C}_{2} \mathrm{OH}_{30} \mathrm{O}_{2}$ & 302 & $7.64 \%$ \\
\hline 32. & trans-Dehydroandrosterone, trifluoroacetate & $\mathrm{C} 21 \mathrm{H} 27 \mathrm{~F} 303$ & 384 & $5.85 \%$ \\
\hline 33. & Androst-5,7-dien-3-ol-17-one & $\mathrm{C}_{19} \mathrm{H}_{26} \mathrm{O}_{2}$ & 286 & $5.17 \%$ \\
\hline 34. & Vitamin A palmitate & $\mathrm{C}_{36} \mathrm{H}_{60} \mathrm{O}_{2}$ & 524 & $4.37 \%$ \\
\hline 35. & Androst-5-en-7-one, 3-(acetyloxy)-, (3ß)- & $\mathrm{C}_{21} \mathrm{H}_{30} \mathrm{O}_{3}$ & 330 & $4.20 \%$ \\
\hline 36. & Dehydroisoandrosterone acetate & $\mathrm{C}_{21} \mathrm{H}_{30} \mathrm{O}_{3}$ & 330 & $4.03 \%$ \\
\hline 37. & $\begin{array}{l}\text { Cyclopropanebutanoic acid, 2-[[2-[[2-[(2- } \\
\text { pentylcyclopropyl)methyl]cyclopropyl]methyl]cyclopropyl]methyl]-, } \\
\text { methyl ester }\end{array}$ & $\mathrm{C}_{25} \mathrm{H}_{42} \mathrm{O}_{2}$ & 374 & $13.7 \%$ \\
\hline 38. & 1-Heptatriacotanol & $\mathrm{C}_{37} \mathrm{H}_{76} \mathrm{O}$ & 536 & $10.5 \%$ \\
\hline 39. & Ursodeoxycholic acid & $\mathrm{C}_{24} \mathrm{H}_{40} \mathrm{O}_{4}$ & 392 & $3.82 \%$ \\
\hline 40. & 9,12,15-Octadecatrienoic acid, 2,3-dihydroxypropyl ester, (Z,Z,Z)- & $\mathrm{C}_{21} \mathrm{H}_{36} \mathrm{O}_{4}$ & 352 & $3.37 \%$ \\
\hline 41. & 6,9,12,15-Docosatetraenoic acid, methyl ester & $\mathrm{C}_{23} \mathrm{H}_{38} \mathrm{O}_{2}$ & 346 & $3.24 \%$ \\
\hline 42. & Androst-5-ene-17-carbonitrile, 4-acetoxy-17-hydroxy- & $\mathrm{C}_{22} \mathrm{H}_{31} \mathrm{NO}_{3}$ & 357 & $2.74 \%$ \\
\hline
\end{tabular}




\begin{tabular}{|c|c|c|c|c|}
\hline 43. & 9,12-Octadecadienoic acid (Z,Z)- & $\mathrm{C}_{18} \mathrm{H}_{32} \mathrm{O}_{2}$ & 280 & $6.65 \%$ \\
\hline 44. & Ethanol, 2-(9,12-octadecadienyloxy)-, (Z,Z)- & $\mathrm{C}_{20} \mathrm{H}_{38} \mathrm{O}_{2}$ & 310 & $5.36 \%$ \\
\hline 45. & 8,11-Octadecadienoic acid, methyl ester & $\mathrm{C}_{19} \mathrm{H}_{34} \mathrm{O}_{2}$ & 294 & $4.52 \%$ \\
\hline 46. & Ethyl 9,12-hexadecadienoate & $\mathrm{C}_{18} \mathrm{H}_{32} \mathrm{O}_{2}$ & 280 & $3.82 \%$ \\
\hline 47. & $\begin{array}{l}\text { Cyclopropaneoctanoic acid, 2-[[2-[(2- } \\
\text { ethylcyclopropyl)methyl]cyclopropyl]methyl]-, methyl ester }\end{array}$ & $\mathrm{C}_{22} \mathrm{H}_{38} \mathrm{O}_{2}$ & 334 & $3.82 \%$ \\
\hline 48. & 9,12-Octadecadienoic acid (Z,Z)-, methyl ester & $\mathrm{C}_{19} \mathrm{H}_{34} \mathrm{O}_{2}$ & 294 & $3.82 \%$ \\
\hline 49. & Podocarp-7-en-3-one, 13ß-methyl-13-vinyl- & $\mathrm{C}_{20} \mathrm{H}_{30} \mathrm{O}$ & 286 & $9.26 \%$ \\
\hline 50. & Pregnan-20-one, 3-(acetyloxy)-5,6-epoxy-, $(3 \beta, 5 \beta, 6 \beta)$ - & $\mathrm{C}_{23} \mathrm{H}_{34} \mathrm{O}_{4}$ & 374 & $9.26 \%$ \\
\hline 51. & $\begin{array}{l}\text { Pregnan-20-one, 5,6-epoxy-3-hydroxy-, }(3 \beta, 5 \alpha, 6 \alpha) \text { - } \\
\text { 1-Phenanthrenecarboxaldehyde, 7-ethenyl- }\end{array}$ & $\mathrm{C}_{21} \mathrm{H}_{32} \mathrm{O}_{3}$ & 332 & $7.09 \%$ \\
\hline 52. & 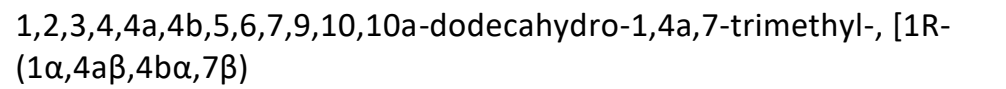 & $\mathrm{C}_{20} \mathrm{H}_{30} \mathrm{O}$ & 286 & $5.01 \%$ \\
\hline 53. & 26,27-Dinorergosta-5,24-dien-3-ol, (3ß)- & $\mathrm{C}_{26} \mathrm{H}_{42} \mathrm{O}$ & 370 & $3.83 \%$ \\
\hline 54. & Pregn-5-en-20-one, 3,17-dihydroxy-, 3-acetate & $\mathrm{C}_{23} \mathrm{H}_{34} \mathrm{O}_{4}$ & 374 & $3.39 \%$ \\
\hline 55. & $\begin{array}{l}\text { Butanoic acid, 1a,2,5,5a,6,9,10,10a-octahydro-5,5a-dihydroxy-4- } \\
\text { (hydroxymethyl)-1,1,7,9-tetramethyl-11-oxo-1H-2,8a- } \\
\text { methanocyclopenta[ }\end{array}$ & $\mathrm{C}_{24} \mathrm{H}_{34} \mathrm{O}_{6}$ & 418 & $8.59 \%$ \\
\hline 56. & $\begin{array}{l}\text { Ethisterone } \\
1 \mathrm{H}-2,8 \mathrm{a}-\text { Methanocyclopenta[a]cyclopropa[e]cyclodecen-11-one, }\end{array}$ & $\mathrm{C}_{21} \mathrm{H}_{28} \mathrm{O}_{2}$ & 312 & $6.58 \%$ \\
\hline 57. & $\begin{array}{l}\text { 1a,2,5,5a,6,9,10,10a-octahydro-5,5a,6-trihydroxy-1,4- } \\
\text { bis(hydroxymethyl)- }\end{array}$ & $\mathrm{C}_{20} \mathrm{H}_{28} \mathrm{O}_{6}$ & 364 & $5.30 \%$ \\
\hline 58. & $\begin{array}{l}\text { Octanoic acid, 1a,2,5,5a,6,9,10,10a-octahydro-5,5a-dihydroxy-4- } \\
\text { (hydroxymethyl)-1,1,7,9-tetramethyl-11-oxo-1H-2,8a- } \\
\text { methanocyclopenta }\end{array}$ & $\mathrm{C}_{28} \mathrm{H}_{42} \mathrm{O}_{6}$ & 474 & $4.89 \%$ \\
\hline 59. & $\begin{array}{l}\text { 2,4,6-Decatrienoic acid, 1a,2,5,5a,6,9,10,10a-octahydro-5,5a- } \\
\text { dihydroxy-4-(hydroxymethyl)-1,1,7,9-tetramethyl-11-oxo-1H-2,8a-;; }\end{array}$ & $\mathrm{C}_{30} \mathrm{H}_{40} \mathrm{O}_{6}$ & 496 & $4.70 \%$ \\
\hline 60. & 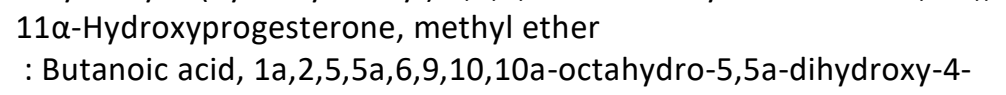 & $\mathrm{C}_{22} \mathrm{H}_{32} \mathrm{O}_{3}$ & 344 & $4.52 \%$ \\
\hline 61. & $\begin{array}{l}\text { (hydroxymethyl)-1,1,7,9-tetramethyl-11-oxo-1H-2,8a- } \\
\text { methanocyclopenta }\end{array}$ & $\mathrm{C}_{24} \mathrm{H}_{34} \mathrm{O}_{6}$ & 418 & $26.5 \%$ \\
\hline 62. & $\begin{array}{l}\text { 5aH-3a,12-Methano-1H- } \\
\text { cyclopropa[5',6']cyclodeca[1',2':1,5]cyclopenta[1,2-d][1,3]dioxol- } \\
\text { 13-one, 1a,2,3,9,12,12a-hexahydro- }\end{array}$ & $\mathrm{C}_{23} \mathrm{H}_{32} \mathrm{O}_{5}$ & 388 & $23.4 \%$ \\
\hline 63. & $\begin{array}{l}\text { 2,4,6-Decatrienoic acid, 1a,2,5,5a,6,9,10,10a-octahydro-5,5a- } \\
\text { dihydroxy-4-(hydroxymethyl)-1,1,7,9-tetramethyl-11-oxo-1H-2,8a }\end{array}$ & $\mathrm{C}_{30} \mathrm{H}_{40} \mathrm{O}_{6}$ & 496 & $11.4 \%$ \\
\hline 64. & 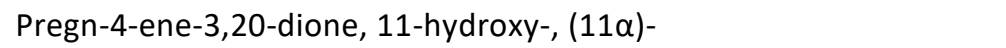 & $\mathrm{C} 21 \mathrm{H} 3003$ & 330 & $2.68 \%$ \\
\hline 65. & 9-Octadecenamide, (Z)- & $\mathrm{C}_{18} \mathrm{H}_{35} \mathrm{NO}$ & 281 & $1.94 \%$ \\
\hline 66. & 16-Allopregnen-3 $\beta$-ol-20-one & $\mathrm{C}_{21} \mathrm{H}_{32} \mathrm{O}_{2}$ & 316 & $1.72 \%$ \\
\hline 67. & Pregn-4-ene-3,6-dione & $\mathrm{C}_{21} \mathrm{H}_{30} \mathrm{O}_{2}$ & 314 & $43.9 \%$ \\
\hline 68. & $\begin{array}{l}\text { Tricyclo[5.4.3.0(1,8)]tetradecan-3-ol-9-one, 4-ethenyl-6-(2- } \\
\text { hydroxyacetoxy)-2,4,7,14-tetramethyl- }\end{array}$ & $\mathrm{C}_{22} \mathrm{H}_{34} \mathrm{O}_{5}$ & 378 & $12.9 \%$ \\
\hline 69. & $\begin{array}{l}\text { 2,6,10,14-Hexadecatetraene, 1-benzyloxy-9-(phenylthio)-3,7,11,15- } \\
\text { tetramethyl- }\end{array}$ & $\mathrm{C}_{33} \mathrm{H}_{44} \mathrm{OS}$ & 488 & $10.9 \%$ \\
\hline 70. & : Retinoic acid, 5,6-epoxy-5,6-dihydro- & $\mathrm{C}_{20} \mathrm{H}_{28} \mathrm{O}_{3}$ & 316 & $3.03 \%$ \\
\hline 71. & $\begin{array}{l}\text { 4-(3,3-Dimethyl-but-1-ynyl)-4-hydroxy-2,6,6-trimethylcyclohex-2- } \\
\text { enone }\end{array}$ & $\mathrm{C}_{15} \mathrm{H}_{22} \mathrm{O}_{2}$ & 234 & $2.44 \%$ \\
\hline 72. & 1-[2-[2-Bromoacetamido]ethyl]hypoxanthine & $\mathrm{C}_{9} \mathrm{H}_{10} \mathrm{BrN5O} \mathrm{O}_{2}$ & 299 & $2.25 \%$ \\
\hline 73. & $\begin{array}{l}\text { 4H-Cyclopropa[5',6']benz[1',2':7,8]azuleno[5,6-b]oxiren-4-one, 8- } \\
\text { (acetyloxy)-1,1a,1b,1c,2a,3,3a,6a,6b,7,8,8a-dodecahydro-3a }\end{array}$ & $\mathrm{C}_{22} \mathrm{H}_{30} \mathrm{O}_{8}$ & 422 & $8.47 \%$ \\
\hline 74. & $\begin{array}{l}\text { Octanoic acid, } 1 \mathrm{a}, 2,5,5 \mathrm{a}, 6,9,10,10 \mathrm{a}-\mathrm{octah} y d r o-5,5 \mathrm{a}-\mathrm{dihydroxy}-4- \\
\text { (hydroxymethyl)-1,1,7,9-tetramethyl-11-oxo-1H-2,8a- } \\
\text { methanocyclopenta }\end{array}$ & $\mathrm{C}_{28} \mathrm{H}_{42} \mathrm{O}_{6}$ & 474 & $7.48 \%$ \\
\hline 75. & $\begin{array}{l}\text { 2,4,6-Decatrienoic acid, 1a,2,5,5a,6,9,10,10a-octahydro-5,5a- } \\
\text { dihydroxy-4-(hydroxymethyl)-1,1,7,9-tetramethyl-11-oxo-1H-2,8a- }\end{array}$ & $\mathrm{C}_{30} \mathrm{H}_{40} \mathrm{O}_{6}$ & 496 & $6.61 \%$ \\
\hline
\end{tabular}


Dodecanoic acid, 1a,2,5,5a,6,9,10,10a-octahydro-5,5a-dihydroxy-4-

76. (hydroxymethyl)-1,1,7,9-tetramethyl-11-oxo-1H-2,8a-

$\mathrm{C}_{32} \mathrm{H}_{50} \mathrm{O}_{6}$

530

$5.84 \%$

methanocyclopenta

77.

$(5 \beta, 13 \alpha) 3 \alpha$-Methoxy-3 $\beta, 19-e p o x y a n d r o s t-8-e n e-7 \alpha, 17 \beta$-diol, 4,4-

dimethyl-

$\mathrm{C}_{22} \mathrm{H}_{34} \mathrm{O}_{4}$

362

$5.62 \%$

78.

Hexadecanoic acid, 1-(hydroxymethyl)-1,2-ethanediyl ester

$\mathrm{C}_{35} \mathrm{H}_{68} \mathrm{O}_{5}$

568

$5.62 \%$

Table 5: GCMS Profiling of water extract of Ricinus root

\begin{tabular}{lllll}
\hline Sl.no. & Compound & Mol.formula & Mol.wt & Prob. \\
\hline 1 & 9-Octadecenamide, (Z)- & $\mathrm{C}_{18} \mathrm{H}_{35} \mathrm{NO}$ & 281 & $76.1 \%$ \\
2 & 9-Octadecenamide & $\mathrm{C}_{18} \mathrm{H}_{35} \mathrm{NO}$ & 281 & $12.0 \%$ \\
3 & Deoxyspergualin & $\mathrm{C}_{17} \mathrm{H}_{37} \mathrm{~N}_{7} \mathrm{O}_{3}$ & 387 & $3.46 \%$ \\
4 & cis-11-Eicosenamide & $\mathrm{C}_{20} \mathrm{H}_{39} \mathrm{NO}$ & 309 & $2.10 \%$ \\
5 & 9-Octadecenamide, 12-hydroxy-, [R-(Z)]- & $\mathrm{C}_{18} \mathrm{H}_{35} \mathrm{NO}_{2}$ & 297 & $1.61 \%$ \\
6 & 13-Docosenamide, (Z)- & $\mathrm{C}_{22} \mathrm{H}_{43} \mathrm{NO}$ & 337 & $1.54 \%$ \\
\hline
\end{tabular}

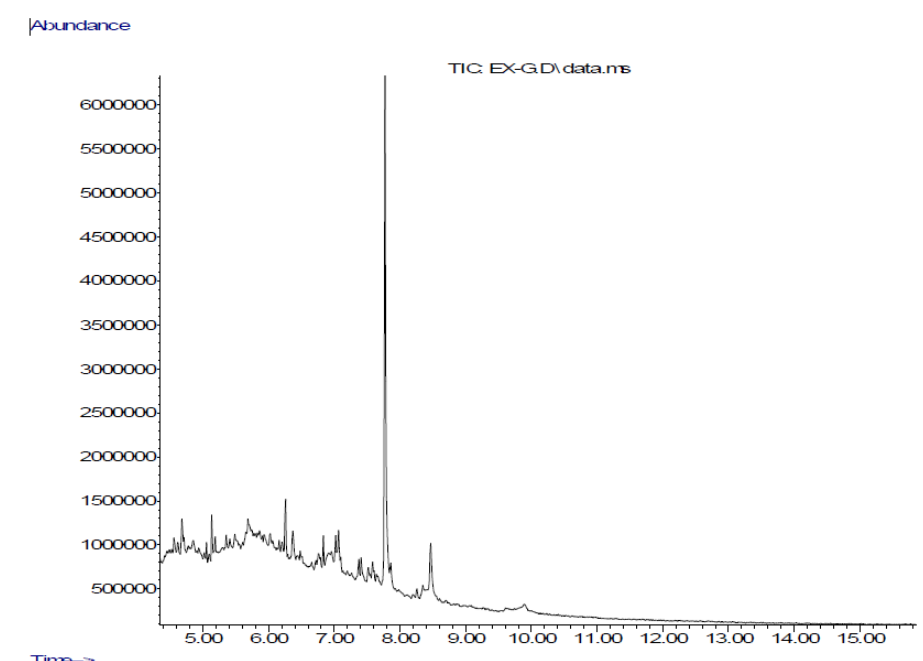

Fig1: GC-Mass Spectrum of the Water extract of Ricinus communis

Abundance

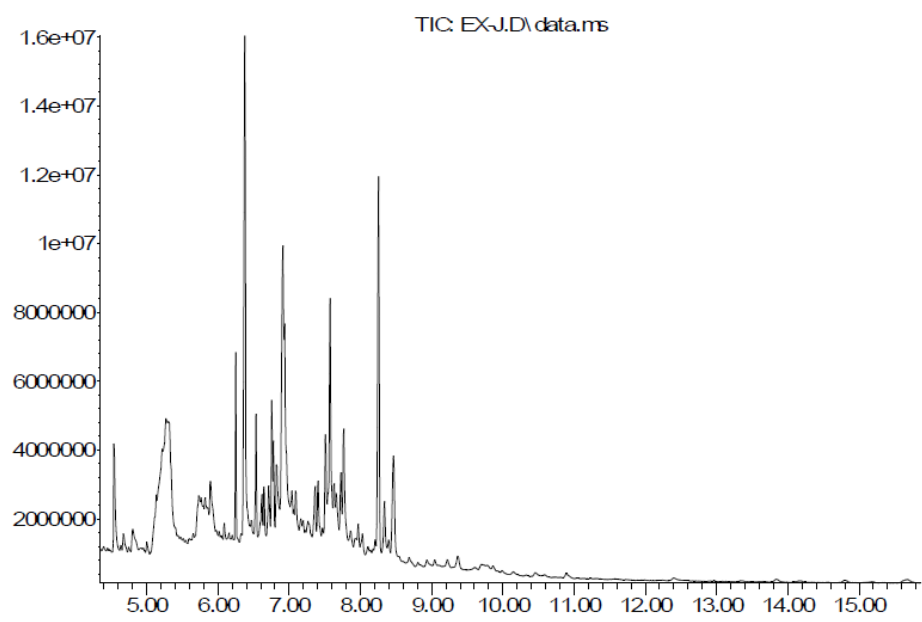

Time $\rightarrow$

Fig2: GC-Mass Spectrum of the methanolic extract of Ricinus communis 


\section{DISCUSSION:}

Analysis outcome of flavonoids and saponins presence in Ricinus communis is comparable to that reported by various authors, using a thin-layer and preparative chromatography analysis. The two toxic alkaloids produced by these major metabolites are sanguinarine and dihydrosanguinarine. Furthermore, main Ricinus communis constituents reported are rutin, gentisic acid, quercetine, gallic acid, kaempferol 3-O-betarutinoside, tannins, ricin $\mathrm{A}, \mathrm{B}$ and $\mathrm{C}^{20}$

\section{CONCLUSION:}

The present study reveals the major pharmacognostic properties of Riccinus. GC-MS profiling of Ricinus communis roots and ash content reveals the amount of inorganic matter present in the crude drug. The phytochemical analysis of Ricinus communis allowed the actual identification of the main secondary metabolites synthesized in roots of the plants using standardized methods.

Therefore, the present work can serve as a source of information for the standardization of the crude drug and a valuable tool for the identification of adulteration.

\section{REFERENCES:}

1. James A. Bourland, Ayodele A. Collins, Scot A. Chester, Sumankalai Ramachandran, and Ronald C. Backer, Determination of Tapentadol(Nucynta) and NDesmethylpentadol in authentic urine specimens by UPLC-Tandem mass spectrometry, Journal of Analytical Toxicology, 2010, 34 : 450457

2. Shankar D. Ved DK, A balanced Perspective for Management of Indian Medicinal Plants" Indian Forester, 2003, 129:275-87.

3. Vijaya Kumar S, Sankar $P$, Varatharajan $R$, Antiinflammatory activity of roots of $A$. aspera. Pharm Biol, 2009, 47:973-5.

4. Handa SS, Indian efforts for Quality Control and Standardization of Herbal Drugs/ products. Proceedings of the $1^{\text {st }}$ Joint Workshop on Quality control and Standardization of Traditional Medicine-indo-China Experience.2004,8-10

5. Ahmad M, Khan MA, Zafar M, Arshad M, Sultana $\mathrm{S}$, Abbasi $\mathrm{BH}$, et al, Use of chemotaxonomic markers for misidentified medicinal plants used in traditional medicines. J Med Plant Res.2010, 4:1244-52.

6. Almeida MR, Flora of Maharashtra. Vol. IV B, Orient Press, Mumbai, 2003, 348.
7. Kirtikar KR, Basu BD. 2nd ed. Dehradun: International Book Distributor; 1985. Euphorbiaceae,

8. Kavita T, Sandhya S, Rajat R, Sanjiv K, Study of phyto-chemical constituents of Ricinus communis Linn. under the influence of industrial effluent Journal of Pharmacy Research, 2013 6:870-873a

9. Anonymous. 1st ed. Part-I. Vol. 1. New Delhi: Govt. of India. Ministry of Health and Family

10. Kensa VM, Syhed YS. Phytochemical screening and antibacterial activity on Ricinus communis $L$. Plant Sciences Feed, 2011, $1: 167-173$

11. Taur DJ, Patil RY, Antiasthmatic activity of Ricinus communis Leaves and Roots. Asian Pacific Journal of Tropical Biomedicine, 2011, 1, S13-S16.

12. Naz R, Bano A, Antimicrobial potential of Ricinus communis leaf extracts in different solvents against pathogenic bacterial and fungal strains. Asian Pacific Journal of Tropical Biomedicine, 2012, 2: 944-947

13. Khursheed R, Naz A, Naz E, Sharif H, Rizwani GH, Antibacterial, antimycelial and phytochemical analysis of Ricinus communis Linn, Trigonella foenum grecum Linn and Delonix regia (Bojer ex Hook.) Raf of Pakistan. Romanian Biotechnological Letters, 2012, 17: 7237- 7244.

14. Jeyaseelan EC, Jashothan PT, In vitro control of Staphylococcus aureus (NCTC 6571) and Escherichia coli (ATCC 25922) by Ricinus communis L. Asian Pacific Journal of Tropical Biomedicine, 2012, 2:717-721

15. Rana M, Dhamija H, Prashar B, Sharma S, Ricinus communis L, A Review.International Journal of PharmTech Research, 2012, 4: 1706-1710.

16. K.R. "Practical Pharmacognosy", Nirali Prakashan, Pune; 9th edition; 2002, 157-158, 2002.

17. Indian Pharmacopoeia, 3rd Edn. Vol. 2, Controller of Publication, Govt. of India, New Delhi, 1985, A88-A90.

18. Indian Pharmacopoeia, Vol.-Il 4th Edition, Controller of Publications, Government of India, New Delhi, 1996, A-47.

19. Welfare, Department of I.S.M. and $H$, the Ayurveda Pharmacopoeia of India. 1999, 34-5.

20. Harborne JB. Berlin: Springer Verlag, Phytochemical methods. A Guide to Modern Techniques of Plant Analysis. Indian Medicinal Plants, 2005, 2274-7.

21. R Pita, A. Anadón and L.M.R Martínez, Ricina: una fitotoxina de uso potencial como arma. Revista de Toxicología, 2004, 21: 51-63. 